\title{
Rapid Disease Progression in a Patient with Advanced NSCLC Harboring a Germline MET Exon 14 Skipping Mutation: A Case Report
}

This article was published in the following Dove Press journal: OncoTargets and Therapy

\author{
Yang Jiao $\mathbb{D D}^{1, *}$ \\ Chen Fang ${ }^{1} *$ \\ Yuchen Yang ${ }^{2}$ \\ Lin Shao ${ }^{2}$ \\ Yi Huang' \\ Qinying Sun' \\ Yuchao Dong (D) \\ 'Pulmonary and Critical Care Medicine, \\ Changhai Hospital, The Naval Medical \\ University, Guangzhou, People's Republic \\ of China; ${ }^{2}$ Burning Rock Biotech, \\ Guangzhou, People's Republic of China \\ *These authors contributed equally to \\ this work
}

Correspondence: Yuchao Dong; Qinying Sun

Pulmonary and Critical Care Medicine, Changhai Hospital, The Naval Medical

University, \#I68 Changhai Road, Yangpu

District, Shanghai, 200433, People's

Republic of China

Email dongyc1020@aliyun.com;

sqy2003I@I63.com

\begin{abstract}
M E T$ exon 14 skipping variants have been identified as a novel type of oncogenic driver mutations in non-small-cell lung cancer (NSCLC), while the germline MET mutation, especially germline $M E T$ exon 14 skipping mutation rarely occurred in NSCLC. Herein, we present the first case of a 33-year-old NSCLC patient with a germline MET exon 14 skipping mutation, who also harbored a somatic EGFR exon 20 insertion. The patient was initially diagnosed with a stage IIB adenosquamous carcinoma. He underwent a thoracoscopic radical resection followed by four cycles of adjuvant chemotherapy but relapsed 2 months after completing the chemotherapy. Afatinib was then prescribed but disease progressed immediately. Subsequently, he received anlotinib but did not respond and died a month later with an overall survival of 9 months. Our case may provide an evidence for the pathogenicity of germline MET exon 14 skipping mutation in NSCLC and suggest it as an adverse prognostic factor.
\end{abstract}

Keywords: $M E T$ exon 14 skipping mutation, rare germline mutation, next-generation sequencing, NGS, lung adenosquamous carcinoma, rapid disease progression

\section{Introduction}

$M E T$ activating mutations have been discovered in a variety of cancer types and demonstrated to play significant roles in tumor initiation, progression, and therapeutic resistance. ${ }^{1}$ Among them, a novel intronic mutation flanking exon 14 in $M E T$, (also known as MET exon 14 skipping mutation) has been identified in NSCLC, which encodes spliced MET transcripts and results in a deletion of the juxtamembrane domain. ${ }^{2,3}$ MET exon 14 skipping mutation is an oncogenic mutation in NSCLC and responds well to MET inhibitors. ${ }^{4}$

Studies have also revealed that MET germline mutations are associated with the development of papillary renal carcinoma (PRC). ${ }^{5,6}$ However, they have rarely been discovered in NSCLC patients and little is known about their association with lung cancer, especially germline MET exon 14 skipping mutation.

Herein, we presented the first case of an NSCLC patient harboring the germline $M E T$ exon 14 skipping mutation, who experienced an exceptional rapid disease progress on the adjuvant chemotherapy and subsequent EGFR TKI afatinib treatment. We present the following case in accordance with the CARE reporting checklist. 


\section{Case Presentation}

A 33-year-old male patient without a smoking history visited the hospital with cough for further treatment. The patient management is described in Figure 1. A chest computed tomography (CT) on August 28, 2019 showed a mass in the upper left lung and enlargement of mediastinal and hilar lymph nodes. No distance metastasis was observed by positron emission tomography/computed tomography (PET/CT). On September 17, 2019, thoracoscopic radical resection of tumor tissue in the left upper lung and mediastinal lymph node dissection of the pulmonary hilum were performed. A Stage IIB (T2N1M0) adenosquamous carcinoma was diagnosed histopathologically. A tumor tissue sample was sent for genetic testing and an EGFR p.S768_D770dup was identified. After surgery, the patient received an adjuvant treatment of albumin-bound paclitaxel and cisplatin for 4 cycles. The following CT scan prompted no sign of recurrence or metastasis. (Figure 1A).

Unfortunately, the patient experienced back pain two months later in March 2020. PET/CT (Figure 1B) showed progressive disease (PD) with an extensive hypermetabolic mass in bilateral lung, extensive hypermetabolic mediastinal and hilar lymph nodes, and bone destruction in the T8, T12 vertebral bone metastasis. A Stage IVB (T4N2M1c) disease was indicated.
The patient was subsequently treated with afatinib (40 mg oral, qd) but quickly developed PD after one month (Figure 1C). Afatinib was then discontinued and a CT-guided lung biopsy was performed. Anlotinib was given as an antiangiogenic therapy while waiting for genetic and pathological results. The pathological result of lung biopsy remains as an adenosquamous carcinoma. Next-generation sequencing (NGS) of the biopsied sample using an 168-gene LungPlasm panel (Burning Rock Biotech, Guangzhou, China) revealed the retaining of EGFR p.S768_D770dup and identified loss of function (LOF) mutations in RBI and TP53 and a MET exon 14 skipping mutation. The $M E T$ exon 14 skipping mutation was subsequently validated as a germline variant $(\mathrm{AF}=49.55 \%)$ by Ugene panel (Burning Rock Biotech, Guangzhou, China) using white blood cells (WBC) (Figure 2). The patient quickly developed PD on bilateral lung metastases revealed by a chest CT (Figure 1D) on May 12, 2020. He died on June 8 due to respiratory failure with an OS of 9 months. All procedures performed in studies involving human participant were in accordance with the ethical standards of the institutional and national research committees, and with the Helsinki Declaration (as revised in 2013). Written informed consent was obtained from the patient.

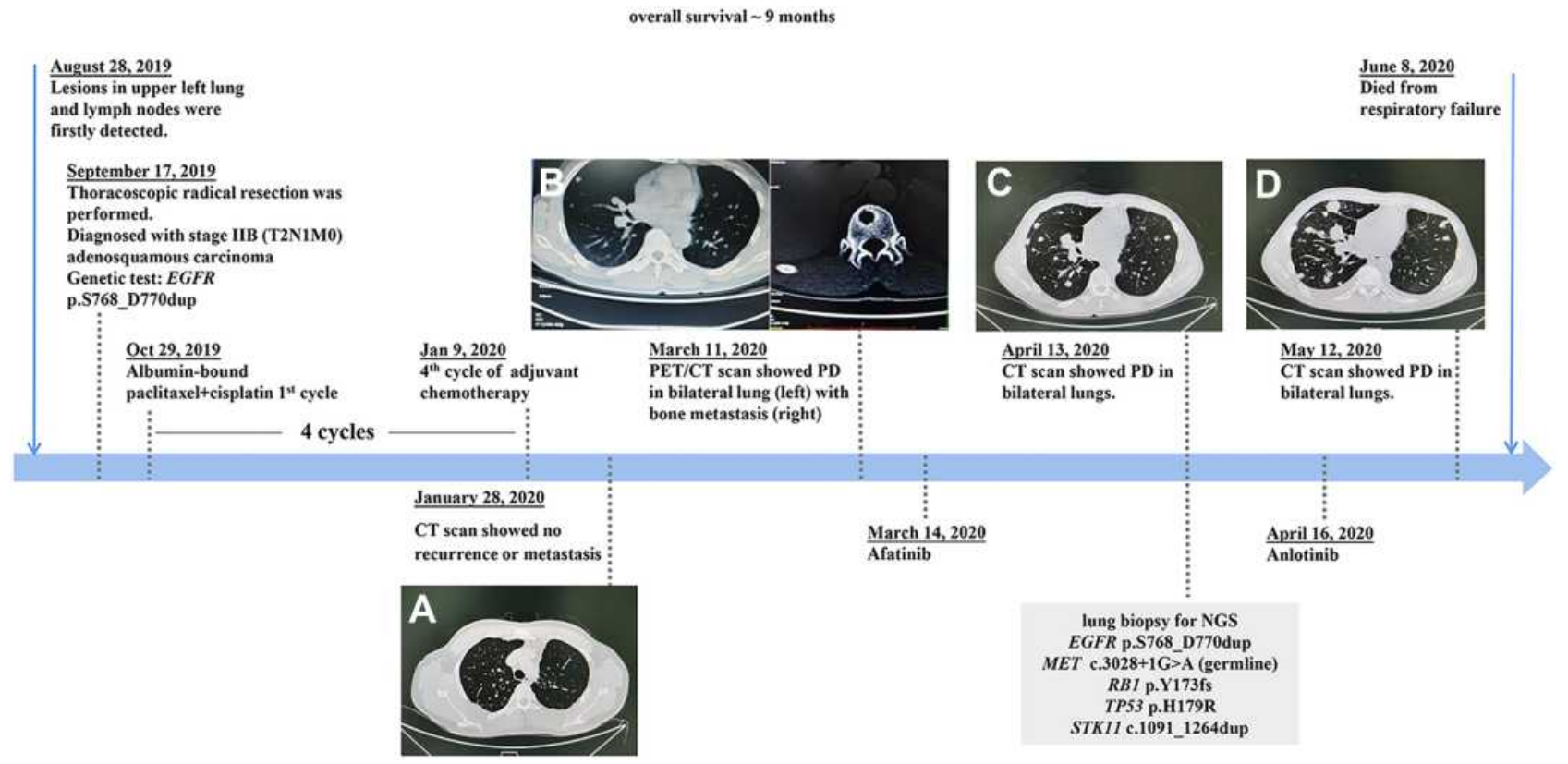

Figure I A summary of patient's treatment history and CT images accordingly. (A) CT scan showed no recurrence or metastasis on Jan 28, 2020. (B) PET/CT scan showed PD in bilateral lung (left) with bone metastasis (right) on Mar II, 2020. (C) CT scan showed PD in bilateral lungs on Apr I3, 2020. (D) CT scan showed PD in bilateral lungs on May 12, 2020.

Abbreviation: PD, progressive disease. 


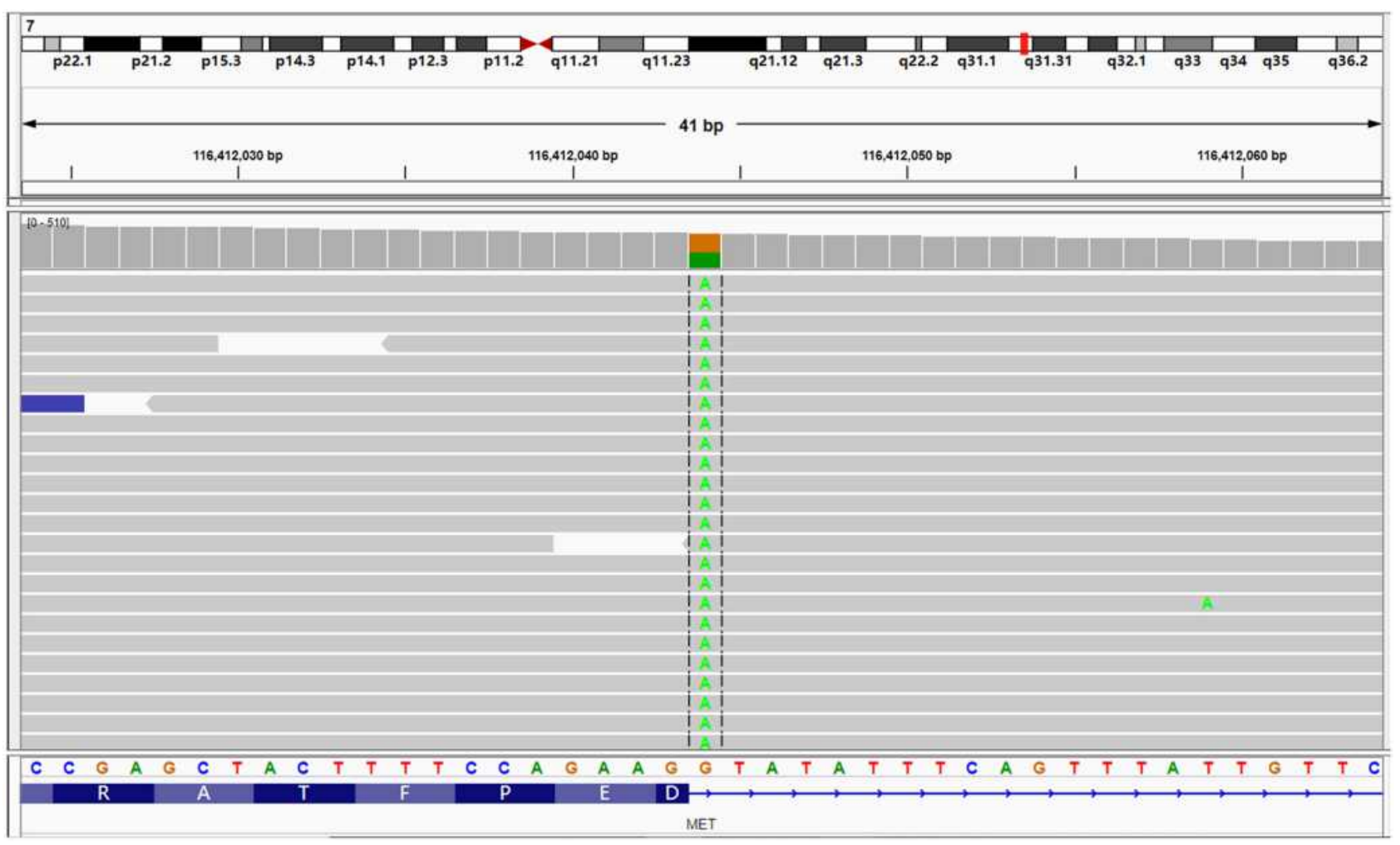

Figure 2 DNA sequencing (with white blood cells) showed a germline MET exon 14 skipping mutation.

\section{Discussion}

To the best of our knowledge, this is the first case where a germline $M E T$ exon 14 skipping mutation was detected in an NSCLC patient. As mentioned above, MET germline mutations are more frequently discovered in hereditary RPCs, which are normally missense and located mainly in the MET kinase domain. ${ }^{5,6}$ Krishnaswamy et $\mathrm{al}^{7}$ has discovered that the majority of MET mutations are germline in lung cancers but still no skipping mutation was detected in this study. Although the patient in our case has no family history of RPC, NSCLC or other types of tumors, he had an early-onset age which might in part suggest the pathogenicity of germline $M E T$ exon 14 skipping mutation.

EGFR exon 20 insertions, including the p.S768_D770dup presented in our case, have been discovered in NSCLC patients, and most of them showed resistance to EGFR TKIs. ${ }^{8}$ Recently, Yang et $\mathrm{al}^{9}$ displayed that the median time to treatment failure (mTTF) of afatinib among 77 patients who harbored EGFR exon 20 insertions is 4.2 months (95\% CI: $2.8-5.3 \mathrm{~m}$ ) and a median duration of response is 11.9 months. However, our case displayed an explosive disease progression to afatinib treatment with only a one-month TTF, which might suggest the presence of additional resistance mechanisms. Krishnaswamy et $\mathrm{al}^{7}$ and other studies have proved that somatic EGFR mutations are not mutually exclusive with mutations in other genes such as MET, TP53 and RB1, which may confer other resistant mechanisms.

MET activating mutation may affect the efficacy of EGFRtargeted therapy in NSCLC patients. Tode et $\mathrm{al}^{10}$ reported a germline MET p.Asn375Lys (c.1125C $>\mathrm{G}$ ) in four affected siblings with EGFR-mutant lung cancer. The study demonstrated that MET and EGFR could mutually compensate for the downstream signaling pathway in vitro and speculated the role of germline MET mutation in the pathogenesis of EGFRmutant lung cancer. In addition, BENEFIT study ${ }^{11}$ has revealed that $E G F R$ mutant NSCLC patients with concomitant oncogenic driver mutations (including MET mutation) showed inferior clinical outcomes to gefitinib treatment. This might in part explain why our case, who harbored the germline MET exon 14 skipping mutation, experienced the rapid disease progression to afatinib treatment.

Besides, synchronous inactivating mutations in $R B 1$ and TP53 were also identified in the patient, which may also affect the treatment efficacy. Recent studies have concluded that a subset of EGFR mutant NSCLC would transform to small cell lung cancer (SCLC) acting as one of the resistance mechanisms to EGFR TKI. ${ }^{12}$ Concurrent $R B 1$ and TP53 inactivating mutations is a molecular characteristic of $\mathrm{SCLC}^{13,14}$ 
and concurrent EGFR/TP53/RB1 triple mutant lung cancers are at unique risk of histologic transformation. ${ }^{15}$ However, although the $E G F R / T P 53 / R B 1$ triple mutant patient reported here progressed on afatinib so fast, no sign of SCLC histologic transformation had been detected pathologically before he died. This might suggest that molecular change happened in advance while the histology remained the same.

Furthermore, MET inhibitor was not available in China at the time when the patient underwent anti-tumor treatments and he died within a short time after the MET exon 14 skipping mutation being identified without targeted therapy. It is known that capmatinib has been subsequently approved by FDA in May 2020, which could have been a potential treatment for this patient.

\section{Conclusion}

In conclusion, we present the first case of an NSCLC patient with a germline $M E T$ exon 14 skipping mutation, who also harbored a somatic EGFR 20 insertion. The patient relapsed rapidly after the surgery and experienced exceptional explosive progression to afatinib treatment. Our study suggests the germline $M E T$ exon 14 skipping mutation might be involved in the pathogenesis of EGFR-mutant-NSCLC and be an adverse prognostic factor. Further investigations are required to validate the findings in our study.

\section{Ethics Approval and Consent to Participate}

The study was approved by the ethical committee of Changhai Hospital and the patient signed an informed consent to participate in the study. Changhai Hospital approved to publish the case details.

\section{Patient Informed Consent}

Written informed consent was obtained for publication of this case report and any accompanying images in an anonymised manner.

\section{Acknowledgments}

Authors thank the patient and patient's kin for agreement to publication of the report. We also thank Yan Chen for her work in case exploration and communication. This work was supported by grants from Shanghai Science and Technology Commission, China [grant number: 19411950402]. The funder did not participate in the study design, study implementation, data collection, data analysis, data interpretation and manuscript writing of the study. Yang Jiao and Chen Fang are co-first authors for this study.

\section{Disclosure}

Y.D. report grants from Shanghai Science and Technology Commission, during the conduct of the study. Authors Y. Y. and L.S. were employed by the company Burning Rock Biotech. The remaining authors declare that the research was conducted in the absence of any commercial or financial relationships that could be construed as a potential conflict of interest.

\section{References}

1. Tovar EA, Graveel CR. MET in human cancer: germline and somatic mutations. Ann Transl Med. 2017;5(10):205. doi:10.21037/atm.20 17.03.64

2. Duplaquet L, Kherrouche Z, Baldacci S, et al. The multiple paths towards MET receptor addiction in cancer. Oncogene. 2018;37 (24):3200-3215. doi:10.1038/s41388-018-0185-4

3. Awad MM, Oxnard GR, Jackman DM, et al. MET exon 14 mutations in Non-small-cell lung cancer are associated with advanced age and stage-dependent MET genomic amplification and c-Met overexpression. J Clin Oncol. 2016. doi:10.1200/JCO.2015.63.4600

4. Drilon A, Clark JW, Weiss J, et al. Antitumor activity of crizotinib in lung cancers harboring a MET exon 14 alteration. Nat Med. 2020;26 (1):47-51. doi:10.1038/s41591-019-0716-8

5. Schmidt L, Duh FM, Chen F, et al. Germline and somatic mutations in the tyrosine kinase domain of the MET proto-oncogene in papillary renal carcinomas. Nat Genet. 1997;16(1):68-73. doi:10.1038/ ng0597-68

6. Salvi A, Marchina E, Benetti A, Grigolato P, De Petro G, Barlati S. Germline and somatic c-met mutations in multifocal/bilateral and sporadic papillary renal carcinomas of selected patients. Int J Oncol. 2008;33(2):271-276.

7. Krishnaswamy S, Kanteti R, Duke-Cohan JS, et al. Ethnic differences and functional analysis of MET mutations in lung cancer. Clin Cancer Res. 2009;15(18):5714-5723. doi:10.1158/1078-0432.CCR-09-0070

8. Wu JY, Wu SG, Yang CH, et al. Lung cancer with epidermal growth factor receptor exon 20 mutations is associated with poor gefitinib treatment response. Clin Cancer Res. 2008;14(15):4877-4882. doi:10.1158/1078-0432.CCR-07-5123

9. Yang JCH, Schuler M, Popat S, et al. Afatinib for the treatment of NSCLC harboring uncommon EGFR mutations: a database of 693 cases. $J$ Thorac Oncol. 2020;15(5):803-815. doi:10.1016/j.jtho.2019. 12.126

10. Tode N, Kikuchi T, Sakakibara T, et al. Exome sequencing deciphers a germline MET mutation in familial epidermal growth factor receptor-mutant lung cancer. Cancer Sci. 2017;108(6):1263-1270. doi: $10.1111 /$ cas. 13233

11. Wang Z, Cheng Y, An T, et al. Detection of EGFR mutations in plasma circulating tumour DNA as a selection criterion for first-line gefitinib treatment in patients with advanced lung adenocarcinoma (BENEFIT): a Phase 2, single-arm, multicentre clinical trial. Lancet Respir Med. 2018;6(9):681-690. doi:10.1016/S2213-2600(18)30264-9

12. Oser MG, Niederst MJ, Sequist LV, Engelman JA. Transformation from non-small-cell lung cancer to small-cell lung cancer: molecular drivers and cells of origin. Lancet Oncol. 2015;16(4):e165-72. doi:10.1016/S1470-2045(14)71180-5

13. George J, Lim JS, Jang SJ, et al. Comprehensive genomic profiles of small cell lung cancer. Nature. 2015;524(7563):47-53. doi:10.1038/ nature 14664 
14. Meder L, König K, Fassunke J, et al. Implementing amplicon-based next generation sequencing in the diagnosis of small cell lung carcinoma metastases. Exp Mol Pathol. 2015;99(3):682-686. doi:10.1016/ j.yexmp.2015.11.002
15. Offin M, Chan JM, Tenet M, et al. Concurrent RB1 and TP53 alterations define a subset of EGFR-mutant lung cancers at risk for histologic transformation and inferior clinical outcomes. $J$ Thorac Oncol. 2019;14(10):1784-1793. doi:10.1016/j.jtho.2019.06.002

\section{Publish your work in this journal}

OncoTargets and Therapy is an international, peer-reviewed, open access journal focusing on the pathological basis of all cancers, potential targets for therapy and treatment protocols employed to improve the management of cancer patients. The journal also focuses on the impact of management programs and new therapeutic

Submit your manuscript here: https://www.dovepress.com/oncotargets-and-therapy-journal agents and protocols on patient perspectives such as quality of life, adherence and satisfaction. The manuscript management system is completely online and includes a very quick and fair peer-review system, which is all easy to use. Visit http://www.dovepress.com/ testimonials.php to read real quotes from published authors. 